\title{
His Bundle Extrasystole or A Dual Atrioventricular Nodal Response
}

\author{
Mohammad Ali Akbarzadeh ${ }^{1,2, *}$ \\ ${ }^{1}$ Cardiovascular Research Center, Shahid Beheshti University of Medical Sciences, \\ Tehran, Iran \\ ${ }^{2}$ Cardiology Department, Shahid Modarres Hospital, Tehran, Iran \\ * Corresponding author: Mohammad Ali Akbarzadeh, Cardiovascular Research \\ Center, Shahid Modarres Hospital, Saadat Abad Street, Tehran, Iran. E-mail: \\ akbarzadehali@yahoo.com
}

DOI: $10.21859 / \mathrm{ijcp}-03104$

Submited: 09-15-2017

Accepted: 11-14-2017

\section{Keywords:}

Tachycardia, Ectopic Junctional

Atrioventricular Nodal

Reentrant Tachycardia

\section{Abstract}

A dual atrioventricular (AV) nodal response and His bundle extrasystole cannot be different in many cases with certainty. We present a 31-year-old man with episodes of palpitation and conducted and non-conducted His bundle extrasystole detected during an electrophysiology study.

(C) 2018. International Journal of

Cardiovascular Practice.

\section{INTRODUCTION}

Hisian extrasystole in the electrophysiology study may be miss-diagnosis as a dual atrioventricular physiology. Differentiating these two patterns is very difficult and their managements are different. When ablation of the slow pathway can perform easily without any significant risk, ablation of His extrasystole may be complicated with complete atrioventricular blockage [1]. In this case report, we present a case of Hisian extrasystole and explain some clues for differentiating these two events.

\section{CASE PRESENTATION}

A 31-year-old man presented with recurrent palpitations and episodes of non-sustained narrow QRS tachycardia which did not resolve by beta blocker and flecainid. His clinical examination, laboratory findings and Doppler echocardiography were normal. During sinus rhythm, the ECG was unremarkable. Due to his significant symptoms, electrophysiological study was performed after discontinuation of the beta blocker and antiarrhythmic medications. A normal sinus rhythm was observed at baseline. (AH interval of $65 \mathrm{~ms}$ and an $\mathrm{HV}$ interval of $58 \mathrm{~ms}$ ). No arrhythmia was induced with programed atrial and ventricular stimulations. After isoproterenol infusion, intracardiac electrogram demonstrated an isolated His signal, followed by ventricular activation (Fig 1 ). What is the mechanism?

\section{DISCUSSION}

At a glance to intracardiac electrogram, the atrial rhythm seems to be sinus. In the first beat after atrial activation, the first His bundle electrograms preceded by ventricular depo- larizations with a normal HV interval, but the second one did not precede by ventricular activation. There were two possible mechanisms for this finding: Hisian extrasystole and 1:2 atrioventricular node conduction (antagrade conduction of atrial rhythm through both fast and slow pathways); the blockage below the His can be explained by the short

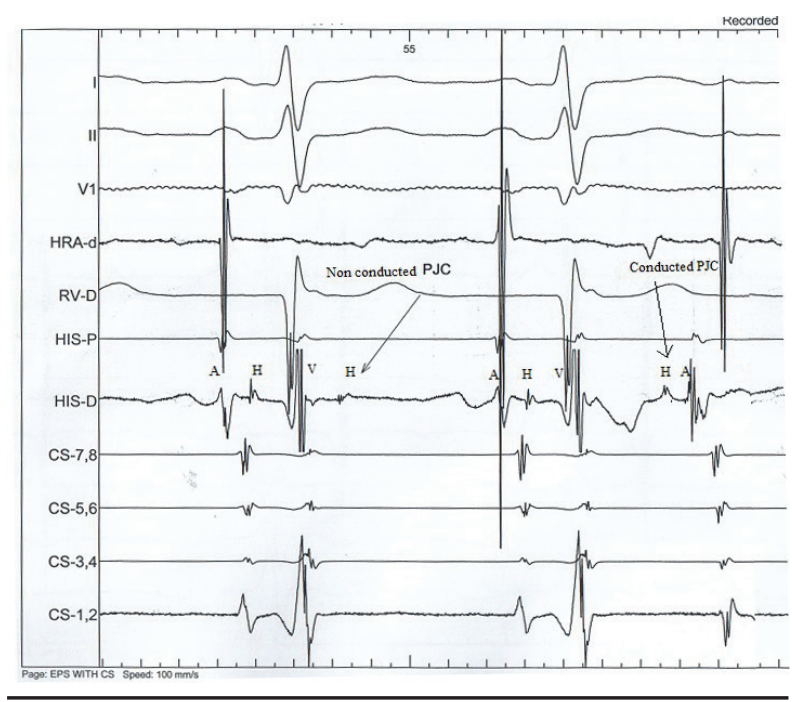

Figure 1: Intracardiac Electrogram Demonstrated Isolated His Signals, followed by Ventricular Activation. The First His Bundle Extrasystole was Blocked but the Second Extrasystole was Followed by Atrial without Ventricular Activation as its more Prolonged Couple Interval from Previous His Depolarization. 
$\mathrm{H}-\mathrm{H}$ intervals preceded after a long-long sequence. A dual $\mathrm{AV}$ nodal response and His bundle extrasystole cannot be different in many cases with certainty, but there are some clues that may suggest the diagnosis. The coupling interval of His activation can vary in both conditions. Although in the case that His extrasystoles originate from the proximal His, the His bundle activation is sequential from proximal to distal as in 1:2 AV conduction, if the extrasystole originates from the distal part, the reverse activation of His can differentiate it from the other condition. Absence of the atrial-His bundle jump during atrial extra-stimulation is also in favor of extrasystole $[2,3]$. During our study, we found some other beats with different conduction patterns. As seen in Fig 1, after the $3^{\text {rd }}$ sinus beat, the second His deflection was followed by atrial without ventricular activation. As there was no His deflection between these atrial and ventricular activations, this means that the impulse from His propagated to atrium (from fast pathway), but was blocked to propagate to the ventricle. This beat also cannot roll out any of the conditions as this beat can be an echo beat or a His extrasystole with retrograde conduction and antagrade block to ventricle, as its more prolonged couple interval from previous His depolarization. This pattern may present at the electrocardiography as a non-conducted premature atrial contraction [4]. Our case was in favor of His extrasystole as the atrial-His bundle jump was absent during atrial extra-stimulation. The patient did not accept the risk of atrioventricular blockage, so ablation was not performed for him.

\section{DISCLOSURES}

Authors declare that they had no conflict of interest.

\section{REFRENCES}

1. Eizmendi I, Almendral J, Hadid C, Ortiz M. Successful catheter cryoablation of Hisian ectopy using 2 new diagnostic criteria based on unipolar and bipolar recordings of the His electrogram. J Cardiovasc Electrophysiol. 2012;23(3):325-9. DOI: 10.1111/j.15408167.2011.02217.x PMID: 22082346

2. Akbarzadeh MA, Fazelifar AF, Bafruee NB. A case of dual atrioventricular nodal nonreentrant tachycardia: An unusual cause of tachycardia-induced cardiomyopathy. J Arrhythm. 2015;31(5):328-30. DOI: 10.1016/i.joa.2015.04.008 PMID: 26550094

3. Hadid C, Di Toro D, Gallino S, Labadet C. Differential diagnosis between dual ventricular response and bigeminy arising from the bundle of His. Rev Esp Cardiol (Engl Ed). 2013;66(6):515. DOI: 10.1016/j.rec.2013.02.003 PMID: 24776068

4. Jastrzebski M, Sondej T, Kukla P. [Concealed His bundle extrasystoles causing atrioventricular block]. Kardiol Pol. 2013;71(4):421-4. DOI: $10.5603 /$ KP.2013.0074 PMID: 23788352 